\title{
Actitudes de la sociedad frente a los Derechos Humanos de las personas en situación de calle que consumen la droga "H", en el cantón la libertad durante el tiempo de Covid-19
}

Society attitudes towards the Human Rights of homeless people who consume the socalled drug "H", in the canton of La Libertad (Ecuador) during Covid-19 times

Francia Cevallos Mora

Andrea Guale Sánchez

Ivette Idrobo Narváez

Gino Apolinario Suárez

Fecha de recepción: 04 de mayo del 2020

Fecha de aceptación: 20 de junio del 2020 


\section{Actitudes de la sociedad frente a los derechos humanos de las personas en situación de calle que consumen la droga "H", en tiempos de Covid-19}

Society attitudes towards the Human Rights of homeless people who consume the so-called drug " $\mathrm{H}$ ", during Covid-19 pandemic

Francia Cevallos Mora ${ }^{1}$. Andrea Guale Sánchez ${ }^{2}$. Ivette Idrobo Narváez ${ }^{3}$. Gino Apolinario Suárez ${ }^{4}$

Como citar: Cevallos, F., Guale, A., Idrovo, I., Apolinario, G., (2020). Actitudes de la sociedad frente a los derechos humanos de las personas en situación de calle que consumen la droga "H", en tiempos de Covid-19, Revista Universidad de Guayaquil. 131(2), 58-67. DOI: https://doi.org/10.53591/rug.v131i2.1368

\section{RESUMEN}

El impacto de la pandemia mundial del COVID-19 cambió profundamente a la sociedad, no solo en el ámbito de salud, sino también en aspectos sociales y de inclusión. En el Ecuador, la Constitución establece los derechos de una integridad personal, sin embargo, es una premisa, que no se cumple para las personas en situación de calle. En el Cantón La Liberad, existe un alto índice de prevalencia de consumo de la droga $\mathrm{H}$, propiciada por las personas que viven en las calles en la zona del Mercado de mariscos -Calle Zona H- autodenominado así por los moradores y transeúntes.

Existe la necesidad de realizar un análisis de las prácticas emprendidas, desde lo local a lo nacional, en atención a este grupo investigado como respuesta a la emergencia sanitaria covid-19. El estudio denota la vulnerabilidad que tienen estos individuos y la falta de acceso a sus derechos, llevándolos a condiciones de pobreza y desempleo, inseguridad, insalubridad, violencia y la dependencia al consumo de alcohol y sustancias nocivas. Finalmente, debe proponerse un sistema de protección integral con un abordaje y atención de todas sus particularidades de manera transversal desde la perspectiva de Derechos Humanos, para otorgarle una vida digna e inclusiva.

\section{Palabras clave:}

Derechos Humanos, COVID-19, Violencia, Drogas, Sociedad, Seres Humanos, Situación Callejera, Ecuador.

' Magister en Dirección de Empresas y Marketing, Universidad Tecnológica Empresarial de Guayaquil. Correo electrónico: francia.cevallos@hotmail.com

${ }^{2}$ Magister en Dirección de Empresas y Marketing. Universidad Tecnológica Empresarial de Guayaquil. Correo Electrónico: gualeandrea@gmail.com

${ }^{3}$ Máster en Relaciones Internacionales, National University of Public Service., Hungría. Correo electrónico: ivetteidrobonarvaez@gmail.com

${ }^{4}$ Ingeniero en Marketing. Universidad Estatal Península de Santa Elena. Correo electrónico: gino_apolinario@ hotmail.com 


\begin{abstract}
The global COVID-19 pandemic has deeply transformed our societies in every aspect, highly impacting the lives of people who live under poverty lines. In Ecuador the scenario has worsened for vulnerable populations, even though the legal Constitution establishes the rights of personal integrity, people living on the streets have been left behind. In the Canton La Libertad, the prevalence rate of the consumption of the so-called drug " $\mathrm{H}$ " has increased due to the use of it by homeless located in the area of the Seafood Market-Calle Zona H.

The need to carry out an analysis of the situation has been identified as a response to the Covid19 health emergency. The study has shown the vulnerability in which they live as well as the lack of access to guarantee of their rights as stated in the Constitution.

This has led them to higher poverty rates and unemployment, instability, lack of access to health services, violence, and problematic dependence on alcohol and harmful substances.

Therefore, this analysis exposes the need of a comprehensive protection system, with an approach and care to all its particularities, developed in a multidimensional way from the Human Rights perspective.
\end{abstract}

\title{
Key words
}

Human Rights, Covid-19, Violence, Drugs, Society, Human Beings, Street Situation, Ecuador.

\section{INTRODUCCIÓN}

El marco del "Sistema de protección especial” en Ecuador, precisa la inclusión y atención a las personas y familias que se encuentran en riesgo o en casos de abandono, pues es claro que la ruta de atención, protección y restitución de los derechos humanos debería ser garantizado en el ámbito local y nacional, incorporando varios enfoques donde el Estado ecuatoriano reconoce el derecho a la integridad personal (Constitución de la República del Ecuador, 2008) y a una vida libre de violencia, no obstante la Sociedad Civil organizada que investiga y se relaciona con la Protección de Derechos ha detectado que en la ejecución de los procesos de atención, protección, restitución y reparación integral de los derechos de la población en situación de calle han presentado situaciones o nudos críticos que entorpecen procesos y hacen que persistan formas variadas de violencia, tales como, situaciones de intolerancia generados espacios privados (familia), en espacios públicos (sociedad), y violación de derechos humanos. 
La crisis sanitaria mundial a inicios de este año ha obligado a mirar las adversidades incluyendo aquellas que forman parte de la política desde sus fundamentos, como la desproporcionalidad de derechos humanos asociada a la violencia hacia las personas en situación de calle, en contexto del toque de queda decretado en nuestro país. En la Ciudad de La Libertad, Provincia de Santa Elena se ha incorporado con cierta fuerza en la agenda política la problemática de las personas en situación de calle. El objetivo de este documento es analizar las prácticas que son emprendidas desde lo local en atención a personas en situación de calle que consumen $\mathrm{H}$, como respuesta a la emergencia sanitaria COVID-19, temática que forma parte de un problema de interés político y social en esta ciudad, así como permitirá identificar la caracterización de convivencia de estas personas. Existe muy poca información sobre estas poblaciones, ya que son personas estigmatizadas por la sociedad y, por lo tanto, son las de más difícil acceso, sin embargo como un complemento al Proyecto sobre la H y Estigma que se ejecutó en el cantón La Libertad de la provincia de Santa Elena (Parametria Consultores, 2020) y por el alto índice de prevalencia de consumo de esta droga, existe la necesidad de realizar un análisis de las prácticas emprendidas desde lo local en atención a personas en situación de calle como respuesta a la emergencia sanitaria covid-19, del cantón La Libertad.

Los resultados responden a un trabajo de campo determinando dos actores principales: Autoridades/ Líderes / Representantes de la localidad que aborden personas en situación de calle, y las personas en situación de calle que se encuentren en el albergue temporal creado por la emergencia sanitaria, mediante una metodología cualitativa: entrevista semi estructurada y grupo focal respectivamente.

\section{La "invisibilidad" de las Personas en situación de calle y sus realidades en época de Pandemia COVID-19}

El contexto internacional refiere la urgencia para abordar una crisis humanitaria desde la perspectiva de los Derechos Humanos y basado en evidencia científica, situación que ha estado invisibilizada por mucho tiempo (Departamento de Comunicación Global Naciones Unidas, 2020). En Ecuador, desde El Estado y la sociedad civil, fundamentan la necesidad de ofrecer nuevas modalidades de atención a las personas en situación de calle y sus entornos, esto debería implicar también la atención a aquellas personas que consumen, abusan o han desarrollado trastornos por consumo de sustancias y que conviven en condiciones deplorables.

El país cuenta con el Ministerio de Salud Pública, quién atiende la problemática actual en conjunto con otras entidades como lo es el Instituto Ecuatoriano de Seguridad Social, mismos que cuentan con el apoyo del Estado, quiénes a través del COE Nacional han optado por medidas preventivas para no propagar el virus declarando en todo el país toque de queda de 
acuerdo a la semaforización de cada cantón y otras medidas necesarias (Servicio Nacional de Gestión de Riesgos y Emergencias, 2020), sin embargo, existe evidencia de que si bien son importantes, no son suficientes, sobre todo para la atención de grupos en condiciones de vulnerabilidad, o con características que no los hace ajustarse a los servicios de atención existentes, refiriéndose de manera específica a las personas que se encuentran en situación de calle, (hombres, mujeres, niños, niñas, adolescentes, trabajadoras sexuales, consumidores de sustancias psicoactivas, personas con discapacidad, migrantes y refugiados).

Acorde a lo señalado anteriormente, se hace indiscutible la necesidad de realizar un análisis sobre la respuesta de las autoridades locales en el abordaje de las personas en situación de calle, pues debe de mantenerse y mejorar el servicio con un enfoque de Reducción de Daños (Velázquez Benítez, Friman Rodríguez, \& González García, 2016), para la atención de una crisis humanitaria, dando un contexto muy significativo a salud pública y seguridad de estas personas.

Los problemas asociados más comunes que se encuentran en las personas en situación de calle (hombres, mujeres, niños, niñas, adolescentes, trabajadoras sexuales, consumidores de sustancias psicoactivas, personas con discapacidad, personas adultas mayores, migrantes y refugiados) son:

Epidemias de salud, muchos de estos seres humanos tienen problemas de salud por tener el sistema inmunológico débil, por factores de alimentación, ambientales y contagios.

Violencia relacionada con las drogas, es común observarlo en sectores donde se concentran personas en situación de calle. En el caso de La Libertad, el alto índice de prevalencia de consumo de la $\mathrm{H}$, hace aún más urgente la atención humanitaria.

Encarcelamiento masivo, estas personas al estar sin ocupación alguna, y bajo efectos de sustancias psicoactivas, y con trastornos por consumo de drogas, desencadena en robos, hurtos, y otros delitos. (UNODC, 2018)

Actitud de la sociedad frente a los Derechos Humanos. Visibilizando lo oculto y la verdadera realidad de las calles.

La problemática de las personas en situación de calle se convirtió en los últimos años en un tema de interés público (Seidmann, Di Iorio, Rigueiral, \& Gueglio Saccone, 2016) y se instaló en las agendas de trabajo de las localidades, en el caso del cantón La Libertad no se generó respuesta sino hasta después de la declaratoria de emergencia sanitaria COVID - 19 por parte de las Autoridades. Este análisis le da interés a los posibles motivos que incidieron para que esto sucediese.

En el estudio realizado, en la entrevista al Lcdo. Marino Restrepo, como representante del CETAD Sembradores de Vida y ahora uno de los líderes del Hogar Temporal El Buen Pastor indica : "Bueno, es una parte de un programa de reducción de riesgo que he estado planeando meses atrás basado más en una parte educativa y orientado a las familias, que se puede hacer desde una parte técnica y científica, también con intervención de otras entidades de las organizaciones civiles que están o quieran participar", pudiendo verificar que se entiende y se analiza la realidad de las calles, visibilizando lo que para muchos está oculto, temas tan sensibles donde la empatía juega un rol muy importante, pues a partir del toque de queda estas personas se encontraban en la llamada zona h, no teniendo donde dormir, dónde y cómo alimentarse y qué a corto tiempo se iban a convertir en peligro para la ciudadanía en sí.

Con fecha 22 de marzo, toma la iniciativa el Municipio de La Libertad en coordinación y 
aceptación por parte del CETAD Sembradores de Vida y la Parroquia El Buen Pastor, para el acogimiento de las personas en situación de calle que deambulaban en los alrededores del mercado \#4 del cantón La Libertad, denominado la Zona H, por el alto índice de consumo en dicho sector. La Iniciativa responde a la emergencia sanitaria COVID-19, declarada a nivel nacional (Presidencia del Ecuador, 2020), pues para las autoridades y la comunidad en general consideran un foco de infección muy alto y riesgoso el que estas personas pueden generar por las condiciones en las que habitan y sobreviven, pudiendo propagar cualquier tipo de contagio inmediato.

El Alcalde del cantón La Libertad Señor Víctor Valdivieso manifestó: "Hoy no los podemos dejar abandonados a su suerte, hoy como Alcalde que quiere también preocuparse por ellos, así que también he pedido apoyo al COE Cantonal recursos para ayudar a estas personas, esto es un problema de salud pública, salud debe intervenir para que se los atienda, esto es una enfermedad, esto es una emergencia no nos podemos quedar cruzados de manos", en razón de este análisis y compromiso político y humano, deciden mediante apoyo institucional llevarlos al nuevo Albergue temporal "Hogar de paso El Buen Pastor" dónde recibieron la atención médica para descartar algún tipo de contagio de virus.

Además, se puede rescatar dentro del análisis, el valioso aporte de la oficina de Justicia y Vigilancia de la Municipalidad, bajo orden de su principal el Coronel Germán Arias y el apoyo en territorio del Sr. Ángel Mejía, quienes hicieron el espacio humanitario mirando los debidos procesos y los derechos de los ciudadanos en condición de calle, con el fin de poder movilizarlos al Albergue.

Es a raíz de la información obtenida tras este importante accionar, que las Autoridades y la comunidad como parte de una sociedad tendrán una relevancia práctica, producto de los posibles hallazgos encontrados en la investigación, permitiendo propuestas de intervención queinviten remirar lo que se ha realizado hasta ahora, introduciendo mejoras y estableciendo nuevos procesos, que contemplen fuertemente las necesidades y problemáticas de las personas en situación de calle, pero no desde la visión del experto sino desde la propia mirada de los involucrados, detectando de esta forma las causas que los mantiene en la calle por tanto tiempo sin adherir a procesos que logren sacarlos de esta realidad. 
Caracterización de la Convivencia de las personas en situación de calle, en época de Pandemia COVID-19

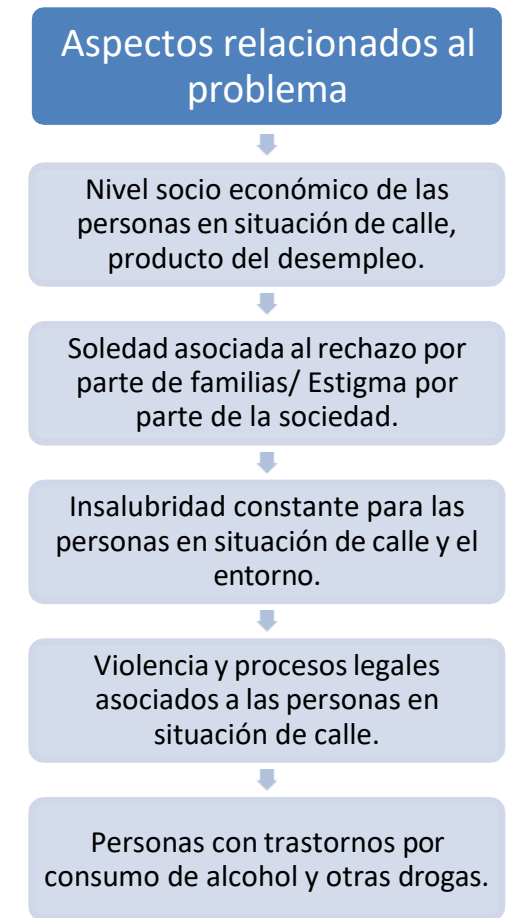

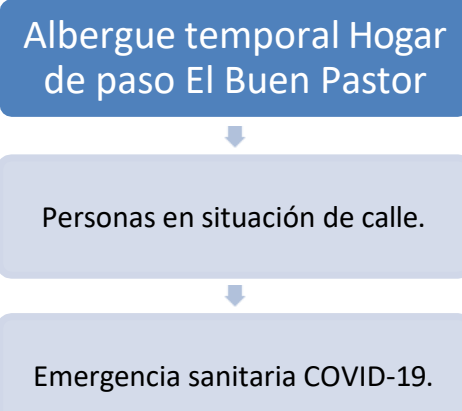

Emergencia sanitaria COVID-19.

Figura 1. Aspectos relacionados al problema (Parametría, 2020)

El momento se da sobre el discurso y cómo a través de las palabras y los significados dados en el intercambio entre el investigador y el investigado, se construye una realidad latente, donde se evidencian las carencias simbólicas y materiales de las personas en situación de calle que dependen de la voluntad de las familias del sector donde deambulan o incluso de lo que logran obtener de manera ilícita en las calles. Por tanto, se ha logrado establecer la caracterización de la convivencia de las personas en situación de calle:

Bajo nivel socio económico, producto del desempleo: La gran mayoría de las personas que viven en situación de calle realizan algún tipo de actividad ilícita relacionada al consumo y/o venta de pequeñas dosis de droga para obtener las suyas, en este caso particular la denominada H (UNODC, 2020), que servirá para aplacar el frío, el hambre, y la verdadera situación de no tener a dónde llegar, la pobreza es en sí misma un problema de derechos humanos urgente que debe abordarse, ya que conlleva a otras violaciones de derechos, viéndose expuestas regularmente a la denegación de su dignidad e igualdad.

Estas personas tropiezan con enormes obstáculos, de índole física, económica, cultural y social, para ejercer sus derechos; en el sector donde normalmente habitan estas personas (Mercado de mariscos, cantón La Libertad -Calle Zona H), los moradores y transeúntes los catalogan como delincuentes, "drogadictos", y mal ejemplo para los jóvenes y la comunidad del sector, por estar sin oficio alguno y deambulando en las calles.

Soledad asociada al rechazo por parte de familias / Estigma por parte de la sociedad: El estudio demuestra que esta población ha sufrido el rechazo por parte de sus padres o familia, inhabilitando a estas personas en las relaciones sociales que pudieran generar un ser 
completamente solo. Así, mismo en el análisis de la información, se puede detectar que estas personas pasan por cambios emocionales que afectan a sus sentimientos, produciendo un miedo extraordinario al juicio negativo de la comunidad, pues a este grupo de personas se los puede ubicar sin ningún problema en una calle del Mercado de Mariscos del cantón La Libertad, dónde los moradores del sector lo denominan la zona de los "Hacheritos", o zona H. Es claro que el estigma que se provoca hacia estas personas empuja a esta población en situación de vulnerabilidad a soportar las relaciones sociales con angustia, desamor y desconfianza.

Insalubridad constante para las personas en situación de calle y el entorno: Al parecer las deplorables condiciones en que se encuentra la calle denominada zona $\mathrm{H}$, en el cantón La Libertad, se debe a que en este sector, se encuentra el mercado de mariscos donde llegan muchas personas en busca de oportunidades de trabajo informal, como venta, limpieza de pescado, entre otros, en donde el olor de los alrededores de estas calles es muy fuerte, debido a la falta de alcantarillado, así el agua de los camiones que desembarcan los mariscos y pescado, principalmente se empoza en determinados lugares porque no tiene por donde drenar.

El agua expulsada por este tipo de situaciones es contaminada y se convierte en un foco infeccioso porque los mosquitos se reproducen con facilidad representando un grave peligro para los habitantes de la zona debido a que están expuestos al contagio de las típicas enfermedades acarreadas por este tipo de insectos como paludismo, dengue clásico y hasta el temido dengue hemorrágico. Sin embargo, existentes alrededor de 80 a 100 personas que han estado viviendo en esta calle acompañados de todas estas situaciones.

Los moradores manifiestan que además de las enfermedades que puedan ser propagadas por razón de las aguas estancadas, este problema en media calle impide el paso vehicular y de los transeúntes, además que proyecta una imagen negativa para la comunidad, Ángel Mejía quién trabaja en Justicia y Vigilancia del Municipio de la Libertad y todos los días están en constante contacto con estas personas, sostiene que "han estado en proceso de acercamiento con diferentes comitivas de la sociedad civil e instituciones públicas para revisar posibles proyectos en ayuda a estas personas y las correspondientes actividades que logren el reparo de esta calle en cuento al problema de esta zona, viéndose la oportunidad de trasladar a una gran parte al Albergue creado temporalmente por la situación", y declaratoria de emergencia sanitaria por COVID-19, pues las epidemias de salud son comunes, muchos de estos seres humanos tienen un sistema frágil, por factores de alimentación, ambientales y contagios. Entre la información más del 50\% de las personas en situación de calle consume la droga denominada $\mathrm{H}$ en el sector, por su precio relativamente económico para estas personas, encontrando paquetes desde 0.25 hasta el dólar o más dependiendo de las dosis.

Violencia y procesos legales asociados a las personas en situación de calle: Es una manifestación de la pobreza pocas veces estudiada en muchos países y en Ecuador. Realidad aún menos conocida es la que en particular vivencian las mujeres víctimas de violencia y los migrantes de otros países que han llegado a nuestro país con la esperanza de oportunidades en especial colombianos y venezolanos. (Ministerio de Relaciones Exteriores y Movilizada Humana, 2020)

En el estudio realizado se analizó el discurso de ocho personas en situación de calle a manera de grupo focal, personas que se acogieron al Albergue Temporal, en razón que vivían en la calle denominada zona $\mathrm{H}$ del cantón La Libertad, durante el momento en que paso la emergencia sanitaria COVID-19, logrando identificar que entre varios conceptos de pobreza, 
exclusión y violencia, se relacionaban a procesos legales que se encuentran estrechamente ligados a la experiencia de éstas personas.

Las condiciones de vida de las personas en situación de calle se ven afectada por cometer delitos contra la salud, robo, hurto y otros delitos desde una perspectiva psicosocial (Di Iorio, 2019), por ejemplo los robos se cometen en el mismo sector del mercado de mariscos, generando peleas callejeras, de este modo la relación entre delincuentes, consumidores de $\mathrm{H} \mathrm{y}$ afectados es a veces tan cercana que ocasiona que muchos de esos hechos no se denuncien y, en consecuencia, no se registren.

El presente estudio refleja y centra la atención en la población en situación de vulnerabilidad que cometen delitos como parte de una problemática social y que afecta a todas las áreas de la vida no sólo de ellos que delinquen y de sus familias, sino a la sociedad en general. Otros moradores del lugar sostienen también que el lugar es sumamente peligroso, los asaltos están a la orden del día por lo que requieren resguardo policial.

Trastornos por consumo de alcohol y otras drogas. - El consumo de alcohol y otras drogas es una consecuencia de convivir en un entorno que genera dolor, discriminación, malestar, exclusión y otras situaciones que empujan a las personas a amortiguar ese dolor con el alcohol y otras drogas. (Ahumada Cortez, Gámez-Medina, \& Valdez Montero, 2017)

La adicción es producto de una ingesta reiterativa de esas sustancias que no es sino otra cosa que la vigencia y el aumento de un entorno doloroso que lastima de manera integral a los sujetos en situación de calle: ausencia de familia, de trabajo, alimentos, seguridad, salud, afecto y sobre todo, rechazo.

\section{CONCLUSIÓN}

En consecuencia y producto de este análisis, se identifican muchas privaciones como las condiciones de trabajo peligrosas, la falta de alimentos nutritivos, el acceso desigual a la justicia, la insalubridad del sector donde viven (calles), la falta de poder político y el limitado acceso a la atención de salud, todo esto acompañado al sufrimiento de estas personas en situación de vulnerabilidad, impidiendo hacer realidad sus derechos y perpetuando su pobreza, creando un círculo vicioso de estigmatización, discriminación, impotencia y exclusión.

\section{Discusión}

En este sentido, se torna imperativo que se active un sistema de protección integral a fin de lograr un abordaje y atención de todas sus particularidades (estatus, etnia, sistema migratorio), logrando identificar en diversas situaciones a los individuos en casos de doble vulnerabilidad, no solo por la Pandemia y una perspectiva estigmatizadora, sino desde su consideración humanitaria y del valor de una vida digna e inclusiva.

Es momento de comenzar a cuestionar nuestros pensamientos, discursos e ideas, para visibilizar a las personas en situación de calle, abramos un espacio a la reflexión y crítica donde nos permitamos trabajar de manera transversal desde la perspectiva de Derechos Humanos, cuestionémonos ¿Qué sucede con aquellos, que además de no tener hogar, no se tienen a sí mismos? ¿Será mejor considerar a la calle un lugar para vivir o para sobrevivir? ¿Podremos ser parte de una sociedad distinta y más justa?

Está en manos de todos los diferentes actores sociales el mejorar las condiciones de vida de las personas que se encuentran en situación de calle, todos y cada uno de nosotros podemos ser actores responsables de comunidades más inclusivas que trabajen por alcanzar los Objetivos 
de Desarrollo Sostenible (ODS) planteados por las naciones para el año 2030 sin dejar a nadie atrás.

\section{REFERENCIAS}

Ahumada Cortez, J. G., Gámez-Medina, M. E., \& Valdez Montero, C. (2017). ELCONSUMO DE ALCOHOL COMO PROBLEMA DE SALUD PÚBLICA. $R a$

Ximhai, 13(2), 13-24. Obtenido de https://www.redalyc.org/pdf/461/46154510001.pdf Asamblea Nacional. (2008). Constitución de la República del Ecuador. Obtenido de https://www.asambleanacional.gob.ec/sites/default/files/documents/old/constitucion_ de_bolsillo.pdf

Departamento de Comunicación Global Naciones Unidas. (2020). Sitio Oficial Naciones

Unidas. Obtenido de Naciones Unidas: Respuesta a la COVID-19:

https://www.un.org/es/coronavirus/articles/proteger-derechos-humanos-coronavirus

Di Iorio, J. (2019). VIVIR EN SITUACIÓN DE CALLE EN CONTEXTOS URBANOS:

SUBJETIVIDADES EN RESISTENCIA. Revista Interamericana de Psicología, 167-

179. Obtenido de

https://journal.sipsych.org/index.php/IJP/article/download/1067/975/3530

Ministerio de Relaciones Exteriores y Movilizada Humana. (2020). Sitio Oficial Cancilleria.

Obtenido de Descargas Sito Oficial Cancilleria: https://www.cancilleria.gob.ec/wpcontent/uploads/2020/09/tenci\%C3\%93n_y_protecci\%C3\%93n_de_la_poblaci\%C3\%

93n_venezolana_2020_-_2021-16sept20-final0812999001600384661.pdf

Parametria Consultores. (2020). Proyecto sobre la H y estigma. La Libertad.

Parametría, C. (2020). Proyecto de Personas en Situación de Calle. Santa Elena: Parametría Consultores Ecuador.

Presidencia del Ecuador. (16 de 03 de 2020). Sitio Oficial Ministerio de Defensa del

Ecuador. Obtenido de Descargas de Ministerio de Defensa:

https://www.defensa.gob.ec/wp-

content/uploads/downloads/2020/03/Decreto_presidencial_No_1017_17-Marzo2020.pdf

Seidmann, S., Di Iorio, J., Rigueiral, G., \& Gueglio Saccone, C. (2016). EL CUIDADO EN PERSONAS EN SITUACIÓN DE CALLE. UNA PERSPECTIVA ÉTICA Y POLÍTICA. ( ),163-172.[fecha de Consulta 2 de Diciem. Anuario de Investigaciones, XXIII, 163-172.

Servicio Nacional de Gestión de Riesgos y Emergencias. (2020). Sitio oficial de Servicio Nacional de Gestión de Riesgos y Emergencias. Obtenido de Sitio Oficial Comunicados SNGRE: https://www.gestionderiesgos.gob.ec/director-del-sngredialogo-con-los-alcaldes-sobre-proceso-de-semaforizacion/ 
UNODC. (2018). Informe Mundial sobre las drogas. Oficina de las Naciones Unidas contra la Droga y el Delito.UNODC. (2020). Informe Mundial sobre las Drogas 2020 de la UNODC: el consumo global aumenta a pesar de que el COVID-19 tiene un impacto de gran alcance en los mercados mundiales de drogas. Viena: Servicio de Información de las Naciones Unidas. Obtenido de https://www.unodc.org/mexicoandcentralamerica/es/webstories/2020/06_26_Informe _Mundial_Drogas_2020.html

Velázquez Benítez, D., Friman Rodríguez, N., \& González García, M. (2016). Programas de reducción de daños en las adicciones, un dilema ético. Correo Científico Médico, 20. 